\section{Kidney Blood Pressure Research}

\title{
Association Between Periodontitis and Low- Grade Albuminuria in Non-Diabetic Adults
}

\author{
Seok Hui Kang Jong Won Park Kyu Hyang Cho Jun Young Do \\ Division of Nephrology, Department of Internal Medicine, Yeungnam University Hospital, Daegu, \\ Republic of Korea
}

\section{Key Words}

Periodontitis $\cdot$ Urinary albumin/creatinine ratio $\cdot$ Metabolic syndrome $\cdot$ Diabetes mellitus

\begin{abstract}
Background/Aims: The objective of the present study was to evaluate the clinical association between periodontitis and a high urinary albumin/creatinine ratio (UACR) in individuals without diabetes mellitus. Methods: Data from the Korean National Health and Nutrition Examination Survey were used for this analysis. A high UACR was defined as UACR $\geq 3.9 \mathrm{mg} / \mathrm{g}$ for men and $\mathrm{UACR} \geq 7.5 \mathrm{mg} / \mathrm{g}$ for women. The WHO community periodontal index (CPI) was used to define periodontitis and assess its severity. Results: The numbers of participants without and with periodontitis were 3,046 and 8,571, respectively. The UACR values were higher in participants with periodontitis than in those without periodontitis. Logistic regression showed that the OR for a high UACR with the presence of periodontitis was $1.14(P=0.044)$ on multivariate analysis. CPI score was positively associated with UACR. Conclusions: Periodontitis was associated with UACR in the non-diabetic participants in this study. Therefore, participants with periodontitis should be closely monitored for UACR, which can function as an early marker for renal injury.
\end{abstract}

(C) 2017 The Author(s)

Published by S. Karger AG, Basel

\section{Introduction}

Periodontitis is characterized by the destruction of periodontal tissues and is a marker for chronic inflammatory condition $[1,2]$. Studies have shown that the prevalence of periodontitis is $>50 \%$ in the general population [4-6]. Recent studies have shown that, beyond being a simple indicator for dental health, periodontitis is associated with several diseases including coronary heart disease, stroke, and rheumatologic diseases and that chronic inflammation seems to play a role in the association between periodontitis and 


\section{Kidney Blood Pressure Research}

these diseases [7, 8]. Recently, many studies have focused on the correlation of periodontitis with various diseases that are associated with chronic inflammation as a pathophysiology.

Albuminuria is a well-known microvascular complication and cardiovascular risk factor for patients with diabetes mellitus (DM) [9]. It is also reported to be independently associated with cardiovascular diseases or mortality in individuals without DM [10]. Recent studies have evaluated the importance of low-grade albuminuria, which is defined as high level within urinary albumin/creatinine ratio (UACR) $<30 \mathrm{mg} / \mathrm{g}$. These studies have shown that a high UACR is associated with mortality and the development of cardiovascular diseases in individuals without DM [10-13]. Many researchers have investigated the factors associated with a high UACR, while other studies investigating new markers of a high UACR are ongoing. A high UACR reveals microvascular injury to the kidney and can be a clinical sign of renal injury due to chronic inflammation. Regarding the association between periodontitis and chronic inflammation, the former may be associated with a high UACR as an indicator of microvascular injury. The objective of the present study was to evaluate the clinical association between periodontitis and a high UACR in individuals without DM.

\section{Patients and Methods}

\section{Study population}

This was a cross-sectional study. Data from the Korean National Health and Nutrition Examination Survey (KNHANES 2012-2014) were used for this analysis. The KNHANES is a nationwide, multi-stage, stratified survey of a representative sample of the South Korean population and is conducted by the Korea Centers for Disease Control and Prevention. There were 23,626 participants in the KNHANES survey. Participants were excluded from the present study if they met the following criteria: data could not be provided for periodontitis $(n=1,397)$ or albuminuria $(n=2,944)$, age<19 years $(n=5,026)$, had micro- or macro- albuminuria (UACR $\geq 30 \mathrm{mg} / \mathrm{g} ; \mathrm{n}=750$ ), or had DM (defined as a self-reported history of DM, fasting glucose $\geq 126 \mathrm{mg} / \mathrm{dL}$, or glycated hemoglobin $A 1 \mathrm{c} \geq 6.5 \%$; $=1,892$ ). A total of 11,617 participants were ultimately included in this study. Ethical approval was obtained from the institutional review board of Yeungnam University Hospital. The board waived the need for informed consent since the subjects' records and information were anonymized and de-identified prior to the analysis.

\section{Study variables}

Clinical and laboratory data collected during the examination included the following: age, sex, serum creatinine level $(\mathrm{mg} / \mathrm{dL})$, body mass index $\left(\mathrm{BMI} ; \mathrm{kg} / \mathrm{m}^{2}\right)$, waist circumference $(\mathrm{WC} ; \mathrm{cm})$, fasting blood glucose $(\mathrm{mg} / \mathrm{dL})$, high-density lipoprotein (HDL) cholesterol level $(\mathrm{mg} / \mathrm{dL})$, triglyceride level $(\mathrm{mg} / \mathrm{dL})$, systolic blood pressure (SBP; mmHg), diastolic blood pressure (DBP; mmHg), smoking status, and alcohol intake.

Height was measured to the nearest $0.1 \mathrm{~cm}$ using a portable stadiometer (Seriter, Bismarck, ND, USA). Weight was measured to the nearest $0.1 \mathrm{~kg}$ using a calibrated balance-beam scale (Giant-150N; Hana, Seoul, Korea). BMI was calculated by dividing weight by the height squared. SBP and DBP were measured using a standard sphygmomanometer with the patient in a sitting position. Three measurements were performed for all subjects at 5-minutes intervals, and the average of the second and third measurements was used in the analysis. WC was measured to the nearest $0.1 \mathrm{~cm}$ in the horizontal plane at the midpoint between the uppermost lateral border of the right iliac crest and the lower rib margin at the end of a normal expiration. Fasting blood glucose, HDL cholesterol, and triglycerides were measured using a colorimetric method (Hitachi Automatic Analyzer 7600; Hitachi, Tokyo, Japan). Serum creatinine levels were measured using a Hitachi Automatic Analyzer (alkaline picrate Jaffé kinetic). The estimated glomerular filtration rate (eGFR) was calculated using the Chronic Kidney Disease Epidemiology Collaboration equation [14].

Urinary albumin and creatinine levels were measured from random samples using a turbidimetric immunoassay and colorimetric methods, respectively, on the Hitachi Automatic Analyzer 7600 (Hitachi). The urinary albumin and creatinine levels were measured at the same laboratory for all surveys. The interassay coefficient of variation for all laboratory work was consistently low $(<3.1 \%)$ for urinary albumin and 


\section{Kidney Blood Pressure Research}

creatinine levels. The UACR was calculated as milligram per gram of creatinine. A high UACR was defined as $\mathrm{UACR} \geq 3.9 \mathrm{mg} / \mathrm{g}$ for men and UACR $\geq 7.5 \mathrm{mg} / \mathrm{g}$ for women as previously defined [11]. Because the definitions of high UACR are inconsistent, we analyzed the data using UACR tertiles. The UACR ranges in the low, middle, and high tertiles were $0.03-2.22 \mathrm{mg} / \mathrm{g}, 2.23-4.36 \mathrm{mg} / \mathrm{g}$, and $4.37-29.92 \mathrm{mg} / \mathrm{g}$ in men, respectively, and $0.04-3.02 \mathrm{mg} / \mathrm{g}, 3.03-6.04 \mathrm{mg} / \mathrm{g}$, and $6.05-29.89 \mathrm{mg} / \mathrm{g}$ in women, respectively.

Patients were classified by smoking status as current smokers, ex-smokers, or non-smokers. Alcohol intake was defined using the Korean definition of "standard drinking" based on the World Health Organization (WHO) classification [15]. Alcohol intake was classified into three categories: abstinence (no alcohol consumption within the last year); moderate drinking (women, 0.1-19.99 g pure alcohol/day; men, 0.1-39.99 g pure alcohol/day); and heavy drinking (women, $\geq 20 \mathrm{~g}$ pure alcohol/day; men, $\geq 40 \mathrm{~g}$ pure alcohol/day).

Metabolic syndrome (MetS) was defined according to Adult Treatment Panel III criteria using the modified cutoff values for Asian populations as suggested by the Asia-Pacific guidelines [16]. Elevated blood glucose was defined as a fasting blood glucose level $\geq 100 \mathrm{mg} / \mathrm{dL}$ or a self-reported history of DM. Elevated blood pressure was defined as systolic and diastolic blood pressure $\geq 130 / 85 \mathrm{mmHg}$ or a self-reported history of hypertension. Low HDL cholesterol level was defined as $<40 \mathrm{mg} / \mathrm{dL}$ in men and $<50 \mathrm{mg} / \mathrm{dL}$ in women. Elevated triglyceride level was defined as serum triglyceride $\geq 150 \mathrm{mg} / \mathrm{dL}$. Abdominal obesity was defined as a WC $>90 \mathrm{~cm}$ in men and $>80 \mathrm{~cm}$ in women. MetS was defined as the presence of $\geq 3$ components of MetS.

The oral examination was performed by trained dentists. The WHO community periodontal index (CPI) was used to define periodontitis and assess its severity as previously described [17]. Six sextants were examined (posterior right maxilla, anterior maxilla, posterior left maxilla, posterior right mandible, anterior mandible, and posterior left mandible). The CPI was scored from 0 to 4: 0 , no bleeding or calculus or no pocket depth $\geq 3.5 \mathrm{~mm} ; 1$, bleeding on probing but no calculus but no pocket depth $\geq 3.5 \mathrm{~mm} ; 2$, supra- or sub-gingival calculus but no pocket depth $\geq 3.5 \mathrm{~mm}$ ); $3,3.5 \leq$ pocket depth $<5.5 \mathrm{~mm}$; and 4, pocket depth $\geq 5.5$ $\mathrm{mm}$. All teeth in each sextant were evaluated, and the highest score was defined as a CPI for each participant. Periodontitis was defined as a CPI $\geq 3$.

\section{Statistical analyses}

The data were analyzed using SPSS (version 19; SPSS, Chicago, IL, USA). The survey was designed using a complex, stratified, multistage probability-sampling model; thus, except for the linear regression analyses, all of the analyses accounted for the complex sampling design effect and appropriate sampling weights of the national survey. Categorical variables are expressed as percentage \pm standard error, while continuous variables are expressed as mean \pm standard error. Since UACR was a non-parametric variable, we transformed it to $\log U A C R$. After the analyses, the logUACR values were exchanged to the UACR values. Pearson's $\chi^{2}$ or Fisher's exact test was used to analyze the categorical variables. For continuous variables, means were compared using Student's $t$-test or one-way analysis of covariance. Logistic regression analyses were used to estimate the odds ratios (OR) and 95\% confidence intervals (CI), which were then used to determine the correlation between periodontitis and a high UACR. Multivariate analyses were adjusted for age, sex, BMI, alcohol intake, smoking habits, fasting blood glucose, eGFR, SBP, DBP, WC, HDL cholesterol, and triglyceride level. These selected confounding factors are well known to be related to metabolic disturbances or renal dysfunction including albuminuria. We included all variables as confounding factors because all can be associated with metabolic disturbances or renal injury. Since sex and the presence of MetS may be important confounding factors for UACR, we performed a subgroup analysis for each. $P$-values $<0.05$ were considered statistically significant.

\section{Results}

\section{Participants' clinical characteristics}

The numbers of participants without and with periodontitis were 3,046 (weighted, $\mathrm{n}=6,622,060$ ) and 8,571 (weighted, $\mathrm{n}=21,239,645$ ), respectively (Table 1). The age, BMI, WC, fasting blood glucose, triglyceride, SBP, and DBP values were higher in participants 


\section{Kidney Blood Pressure Research}

Kidney Blood Press Res 2017;42:338-346

\begin{tabular}{|l|l|l|l|l}
\hline DOI: 10.1159/000477784 & (C) 2017 The Author(s). Published by S. Karger AG, Basel
\end{tabular}

Published online: June 08, 2017 www.karger.com/kb with than in those without periodontitis. The eGFR and HDL cholesterol levels were higher in participants without than in those with periodontitis. The proportion of current smokers was greater in the participants with than in those without periodontitis.

\section{Association bet- ween UACR and pe- riodontitis \\ The UACR values} were higher in participants with than in those without periodontitis (participants with periodontitis, 3.78 \pm 1.03 ; participants without periodontitis, 3.28 $\pm 1.02 ; \quad P<0.001$ ). In the multivariate analysis adjusted for confounding factors, the UACR values were higher in participants with periodontitis than in those without pe-

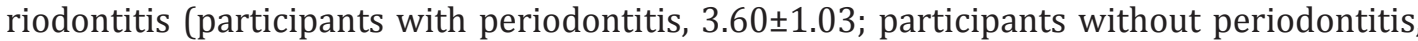
$3.35 \pm 1.02 ; P=0.007)$.

The proportions of participants with a high UACR without and with periodontitis were $27.8 \pm 0.7 \%$ and $38.8 \pm 1.2 \%$, respectively $(P<0.001)$. Logistic regression showed that the OR for a high UACR with the presence of periodontitis was 1.65 (95\% CI, 1.48-1.84; $P<0.001)$ on univariate analysis and $1.14(95 \% \mathrm{CI}, 1.00-1.29 ; P=0.044)$ on multivariate analysis.

The proportion of participants with periodontitis in the low, middle, or high UACR tertiles was $19.7 \pm 0.9 \%, 23.1 \pm 0.9 \%$, and $28.9 \pm 1.0 \%$, respectively. As UACR tertile increased, the proportions of participants with periodontitis increased $(P<0.001)$. On univariate analysis, the OR for the presence of periodontitis was $1.23(95 \% \mathrm{CI}, 1.09-1.39 ; P=0.001)$ for the middle UACR tertile and 1.66 (95\% CI, 1.46-1.88; $P<0.001)$ for the high UACR tertile. On multivariate analysis, the OR for the presence of periodontitis was 1.20 (95\% CI, 1.05-1.38; $P=0.009$ ) for the middle UACR tertile and 1.18 (95\% CI, 1.02-1.36; $P=0.029)$ for the high UACR tertile.

On univariate analysis, the UACR values for CPI score were $3.25 \pm 1.02 \mathrm{mg} / \mathrm{g}$ in 0 , $3.22 \pm 1.05 \mathrm{mg} / \mathrm{g}$ in $1,3.32 \pm 1.02 \mathrm{mg} / \mathrm{g}$ in $2,3.67 \pm 1.03 \mathrm{mg} / \mathrm{g}$ in 3 , and $4.10 \pm 1.04 \mathrm{mg} / \mathrm{g}$ in 4, respectively (Fig. 1; $P=0.903$ for a CPI score of 0 vs 1 ; $P=0.485$ for a CPI score of 0 vs 2; $P<0.001$ for a CPI score of 0 vs $3 ; P<0.001$ for a CPI score of 0 vs $4 ; P=0.589$ for a CPI score of 1 vs 2 ; $P=0.008$ for a CPI score of 1 vs $3 ; P<0.001$ for a CPI score of 1 vs 4 ; $P=0.001$ for a CPI score of 2 vs 3 ; $P<0.001$ for a CPI score of 2 vs $4 ; P=0.030$ for a CPI score of 3 vs 4 ). On multivariate analysis, the UACR values for CPI score were $3.32 \pm 1.03 \mathrm{mg} / \mathrm{g}$ in $0,3.32 \pm 1.05$ $\mathrm{mg} / \mathrm{g}$ in $1,3.39 \pm 1.03 \mathrm{mg} / \mathrm{g}$ in $2,3.53 \pm 1.03 \mathrm{mg} / \mathrm{g}$ in 3 , and $3.90 \pm 1.05 \mathrm{mg} / \mathrm{g}$ in 4 , respectively $(P=0.974$ for a CPI score of 0 vs $1 ; P=0.516$ for a CPI score of 0 vs $2 ; P=0.091$ for a CPI score of 0 vs 3 ; $P=0.001$ for a CPI score of 0 vs $4 ; P=0.667$ for a CPI score of 1 vs $2 ; P=0.232$ for a CPI

\begin{tabular}{lccc} 
Table 1. Participants' clinical characteristics & & \\
\hline Variable & $\begin{array}{c}\text { Periodontitis } \\
(-)\end{array}$ & $\begin{array}{c}\text { Periodontitis } \\
(+)\end{array}$ & $\begin{array}{c}P \text { - } \\
\text { value* }^{*}\end{array}$ \\
\hline Age (years) & $40.4 \pm 0.2$ & $52.2 \pm 0.3$ & $<0.001$ \\
Sex (male, \%) & $49.3 \pm 0.6$ & $61.5 \pm 0.9$ & $<0.001$ \\
Body mass index (kg/m²) & $23.39 \pm$ & $24.06 \pm$ & $<0.001$ \\
Waist circumference (cm) & 0.05 & 0.07 & \\
Fasting blood glucose (mg/dL) & $79.3 \pm 0.2$ & $82.6 \pm 0.2$ & $<0.001$ \\
Triglycerides (mg/dL) & $92.2 \pm 0.1$ & $95.7 \pm 0.2$ & $<0.001$ \\
High-density lipoprotein & $123.0 \pm 1.4$ & $148.1 \pm 2.6$ & $<0.001$ \\
cholesterol (mg/dL) & $53.6 \pm 0.2$ & $50.1 \pm 0.3$ & $<0.001$ \\
Systolic blood pressure (mmHg) & $114.0 \pm 0.2$ & $119.2 \pm 0.4$ & $<0.001$ \\
Diastolic blood pressure (mmHg) & $74.6 \pm 0.2$ & $77.0 \pm 0.3$ & $<0.001$ \\
Alcohol intake (\%) & & & $<0.001$ \\
$\quad$ Abstinence & $20.0 \pm 0.5$ & $25.0 \pm 1.0$ & \\
$\quad$ Moderate & $72.1 \pm 0.6$ & $63.6 \pm 1.1$ & \\
$\quad$ Heavy & $4.1 \pm 0.3$ & $6.1 \pm 0.6$ & \\
$\quad$ Unknown & $3.8 \pm 0.3$ & $5.3 \pm 0.5$ & \\
Smoking habits (\%) & & & $<0.001$ \\
$\quad$ Non-smoker & $57.8 \pm 0.7$ & $41.3 \pm 1.1$ & \\
Ex-smoker & $17.0 \pm 0.5$ & $22.4 \pm 0.9$ & \\
Current smoker & $21.5 \pm 0.6$ & $31.0 \pm 1.1$ & \\
$\quad$ Unknown & $3.7 \pm 0.3$ & $5.3 \pm 0.5$ & \\
eGFR (mL/min/1.73 $\mathrm{m}^{2}$ ) & $100.9 \pm 0.2$ & $92.9 \pm 0.3$ & $<0.001$ \\
\hline
\end{tabular}

The data are expressed as percentages \pm standard errors for categorica
variables and as mean \pm standard errors for continuous variables. ${ }^{*} \mathrm{P}$ values were tested using Student's t-test for continuous variables and eGFR $\chi^{2}$ or Fisher's exact test for catego 


\section{Kidney Blood Pressure Research}

Fig. 1. UACR by CPI score. $* P<0.05$ vs CPI score 0,1 , or 2 ; ${ }^{\#} P<0.05$ vs CPI score $0,1,2$, or 3 . The data are expressed as mean and standard error. Multivariate analyses were adjusted for age, sex, body mass index, alcohol intake, smoking habits, fasting blood glucose, estimated glomerular filtration rate, systolic blood pressure, diastolic blood pressure, waist circumference, high-density lipoprotein cholesterol, and triglyceride level. Abbreviations: CPI, community periodontal index; UACR, urinary albumin/creatinine ratio.

Fig. 2. The UACR value by sex or the presence of MetS. For men without MetS, the mean UACR values in participants without and with periodontitis were $3.00 \pm 1.02$ and $3.97 \pm 1.04$, respectively $(P<0.001)$. For men with MetS, those in participants without and with periodontitis were $5.87 \pm 1.06$ and $8.17 \pm 1.07$ $(P<0.001)$. For women without MetS, the mean UACR values in participants without and with periodontitis were $4.22 \pm 1.02$ and $4.85 \pm 1.05$, respectively $(P=0.004)$. For women with MetS, those in participants without and with periodontitis were $8.04 \pm 1.04$ and $10.38 \pm 1.06$, respectively $(P<0.001)$. The data are expressed as mean and standard error. Abbreviations: MetS, metabolic syndrome; UACR, urinary albumin/creatinine ratio.

score of 1 vs $3 ; P=0.006$ for a CPI score of 1 vs $4 ; P=0.244$ for a CPI score of 2 vs $3 ; P=0.001$ for a CPI score of 2 vs $4 ; P=0.028$ for a CPI score of 3 vs 4). In our study, a CPI score of 3 or 4 on univariate analysis or 4 on multivariate analysis was associated with UACR. Participants with a high CPI score $(\geq 3)$ were associated with an increase in UACR.

\section{Subgroup analyses according to sex and the presence of MetS}

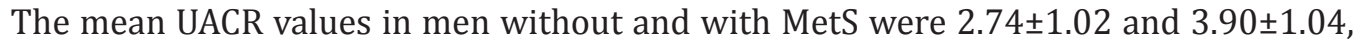
respectively $(P<0.001)$. The mean UACR values in women without and with MetS were $3.67 \pm 1.02$ and $5.26 \pm 1.04$, respectively $(P<0.001)$. For both sexes, UACR was greater in participants with periodontitis than in those without periodontitis, regardless of the presence of MetS (Fig. 2).

For participants without MetS, the proportions of participants with a high UACR without and with periodontitis were $33.0 \pm 1.0 \%$ and $42.5 \pm 1.7 \%$ in men and $20.6 \pm 0.7 \%$ and $25.8 \pm 1.8 \%$ in women, respectively $(P<0.001$ for men and $P=0.004$ for women). For participants with MetS, the proportions of participants with a high UACR without and with periodontitis were 


\section{Kidney Blood Pressure Research}

$49.7 \pm 2.2 \%$ and $58.1 \pm 2.3 \%$ in men and $38.0 \pm 1.6 \%$ and $49.2 \pm 2.5 \%$ in women, respectively $(P=0.006$ for men and $P<0.001$ for women). For both sexes, the proportions of participants with a high UACR were greater in those with than in those without periodontitis, regardless of the presence of MetS.

\section{Discussion}

The present study included only non-DM participants. Periodontitis was associated with the proportion of participants with a high UACR as a categorical variable, while CPI score was positively associated with UACR as a continuous variable. A CPI score of 3 or 4 on univariate analysis or 4 on multivariate analysis was positively associated with an increase in UACR. For both sexes, UACR was greater in participants with than in those without periodontitis regardless of the presence of MetS. In addition, for both sexes, the proportions of participants with a high UACR were also greater in those with than in those without periodontitis, regardless of the presence of MetS.

Periodontitis is reportedly associated with systemic disease. Studies have shown that periodontitis is associated with an imbalance in inflammatory responses, which include immune function, neutrophil activity, and the production of various cytokines [18]. Therefore, periodontitis is recognized as an increased inflammatory status that results in vascular endothelial dysfunction and abnormal lipid metabolism $[19,20]$. Seinost et al. evaluated the association between periodontitis and subclinical flow-mediated dilation of the brachial artery [21]. They showed that periodontitis was associated with lower flowmediated dilation, which reveals endothelial dysfunction, and that the treatment of severe periodontitis reversed this pathological change. Many studies have also shown positive associations between periodontitis and cardiovascular disease or mortality in various populations [22-25].

In a population-based study that included participants with stage 3 and 4 chronic kidney disease who did not undergo renal replacement therapy, a significant association was found between periodontitis and clinical outcomes in non-dialysis patients [26]. An association between periodontitis and clinical outcomes including mortality was also observed in studies of participants on renal replacement therapy $[25,27,28]$. Han et al. analyzed KNHANES data and showed that UACR was associated with periodontitis in DM participants [29]. In their study, micro- or macro-albuminuria, which was defined as a UACR $\geq 30 \mathrm{mg} / \mathrm{g}$, was associated with periodontitis. However, no statistically significance was observed between albuminuria within the normal range and periodontitis. The reasons for this discrepancy may be threefold. First, they enrolled only participants with DM. DM per se is an important inducer of lowgrade albuminuria and may be associated with inflammation above periodontitis. Second, in their study, cutoff values of high UACR were identical for both sexes. The cutoff value for a high UACR is generally higher in women than in men. Third, sample size was smaller in their study $(n=547)$ than in our study $(n=11,617)$ and their analytic method may be deemed as inappropriate in the context of the study. A large number of participants can induce more statistical significance than a small number of participants. In addition, the KNHANES is a nationwide, multi-stage, and stratified survey of a representative sample of the South Korean population. Therefore, the KNHANES provides specific guidelines for analysis and reporting of sample weights. Design stratification and clustering should be considered for analyses using the dataset [30]. However, Han's study did not consider complex survey design.

The aim of our study was to evaluate the association between periodontitis as a trigger of low-grade inflammation and high UACR within the normal albuminuria range $(<30 \mathrm{mg} / \mathrm{g})$ in the non-diabetic population, and we excluded the participants with micro- or macroalbuminuria. Although evidence supports the contribution of periodontitis to triggering systemic inflammation, patients with micro- or macro-albuminuria have more advanced disease than those with a high UACR and albuminuria within the normal range. Patients with 


\section{Kidney Blood Pressure Research}

micro- or macro-albuminuria may be more vulnerable to chronic kidney injuries associated with other cardio-metabolic factors such as blood pressure and dyslipidemia, which are well known as classical triggers of inflammatory status rather than periodontitis. In addition, participants with various cardiometabolic risk factors would be strongly associated with the development of periodontitis, but it is difficult to distinguish whether albuminuria is caused by a primary effect of periodontitis or an indirect effect of periodontitis combined with various cardiometabolic factors.

Studies have investigated the association between periodontitis and UACR in participants with DM or between periodontitis and various clinical outcomes in participants with baseline chronic kidney disease. However, to the best of our knowledge, few studies have examined the association between periodontitis and low-grade albuminuria in participants without DM. Low-grade albuminuria as an early indicator of endothelial and/ or renal injury was the main outcome of our study. We have completely exclude participants with DM since albuminuria and/or UACR can be largely influenced by DM regardless of the control of DM. Our study conclusively showed the associations between periodontitis and UACR as a continuous variable or the proportion of participants with a high UACR as a categorical variable in a general population without DM. In addition, the correlation between insulin resistance and periodontitis is well established, and sex can create significant bias. Therefore, we performed subgroup analyses according to sex and the presence of MetS. Our results showed that regardless of sex and the presence of MetS, periodontitis was associated with greater UACR and proportion of participants with a high UACR.

Definitions of high UACR are inconsistent. Previous studies defined high normal albuminuria as UACR values in the highest tertile, the highest quartile, or above the median level $[11,31,32]$. In their Western population, Arnlöv et al. defined high normal albuminuria as a UACR value $\geq 3.9 \mathrm{mg} / \mathrm{g}$ for men and UACR $\geq 7.5 \mathrm{mg} / \mathrm{g}$ for women using median values [11]. Our study defined high UACR using their cutoff values, but median UACR values in our study were $3.12(0.03-29.92) \mathrm{mg} / \mathrm{g}$ in men and $4.26(0.04-29.89) \mathrm{mg} / \mathrm{g}$ in women. This difference may be associated with the ethnicity and characteristics of the enrolled population. Therefore, we conducted further analysis using UACR tertile. As UACR tertile increased, the proportions of participants with periodontitis increased.

In our study, the UACRs in CPI scores of 0 and 4 were $3.32 \pm 1.03 \mathrm{mg} / \mathrm{g}$ and $3.90 \pm 1.05 \mathrm{mg} / \mathrm{g}$, respectively. Although statistical significance was observed, differences in UACR according to CPI score were small between the CPI scores of 0 and 4 (approximately $0.6 \mathrm{mg} / \mathrm{g}$ ). A larger number of participants may lead to statistical significance. Whether these differences have clinical significance is unclear. Only few studies have investigated cutoff values of high UACR with clinical significance for low-grade albuminuria. Further investigations are needed to identify cutoff values with clinical significance for low-grade albuminuria.

Renal function would be associated with the presence of UACR, and the presence of chronic kidney disease would be a confounding factor in the relationship between periodontitis and UACR. A total of 177 (1.5\%) of the participants had an eGFR<60 mL/ $\mathrm{min} / 1.73 \mathrm{~m}^{2}$, while only one had an eGFR $<30 \mathrm{~mL} / \mathrm{min} / 1.73 \mathrm{~m}^{2}$. Here we did not exclude participants with decreased renal function since their small number would not greatly affect the results. We also added the eGFR as a covariate in the multivariate analyses to reduce the effect of decreased renal function.

This study had several limitations. First, it was retrospective. We could not evaluate the causality between periodontitis and UACR and did not consider ethnic differences. All participants were drawn from the Korean population. Second, we used a single urine spot sample to calculate the UACR. A prospective multi-ethnic study, including accurate parameters such as UACR calculated using a 24-hour urine sample, is warranted to overcome these limitations. 


\section{Kidney \\ Blood Pressure Research}

Kidney Blood Press Res 2017;42:338-346
\begin{tabular}{l|l}
\hline DOI: 10.1159/000477784 & $\begin{array}{l}\text { ( ) 2017 The Author(s). Published by S. Karger AG, Basel } \\
\text { www.karger.com/kbr }\end{array}$ \\
Published online: June 08, 2017 &
\end{tabular}

Kang et al.: Periodontitis and High UACR1

\section{Conclusion}

Periodontitis was associated with UACR in the non-diabetic participants in this study. Therefore, participants with periodontitis should be closely monitored for UACR, which can function as an early marker for renal injury.

\section{Disclosure Statement}

The authors declare that they have no conflict of interests.

\section{Acknowledgements}

This work was supported by the 2014 Yeungnam University Research Grant.

\section{References}

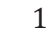

$>2$

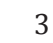

$>4$

$>5$

$>6$

$>7$

$>8$

$>9$

Löe H: Periodontal disease. The sixth complication of diabetes mellitus. Diabetes Care 1993;16:329-334.

Armitage GC: Development of a classification system for periodontal diseases and conditions. Ann Periodontol 1999;4:1-6.

Highfield J: Diagnosis and classification of periodontal disease. Aust Dent J 2009;54:S11-S26.

Eke PI, Dye BA, Wei L, Thornton-Evans GO, Genco RJ: Prevalence of periodontitis in adults in the United States: 2009 and 2010. J Dent Res 2012;91:914-920.

Zhang Q, Li Z, Wang C, Shen T, Yang Y, Chotivichien S, Wang L: Prevalence and predictors for periodontitis among adults in China, 2010. Glob Health Action 2014;7:24503.

Schützhold S, Kocher T, Biffar R, Hoffman T, Schmidt CO, Micheelis W, Jordan R, Holtfreter B: Changes in prevalence of periodontitis in two German population-based studies. J Clin Periodontol 2015;42:121-130. Southerland JH, Taylor GW, Moss K, Beck JD, Offenbacher S: Commonality in chronic inflammatory diseases: periodontitis, diabetes, and coronary artery disease. Periodontol 2000 2006;40:130-143.

Otomo-Corgel J, Pucher JJ, Rethman MP, Reynolds MA: State of the science: chronic periodontitis and systemic health. J Evid Based Den Pract 2012;12:S20-S28.

American Diabetes Association: Standards of medical care in diabetes-2015. Diabetes Care 2016;39:S1S112.

Lioudaki E, Florentin M, Ganotakis ES, Mikhailidis DP: Microalbuminuria: a neglected cardiovascular risk factor in non-diabetic individuals? Curr Pharm Des 2013;19:4964-4980.

1 Arnlöv J, Evans JC, Meigs JB, Wang TJ, Fox CS, Levy D, Benjamin EJ, D’Agostino RB, Vasan RS: Low-grade albuminuria and incidence of cardiovascular disease events in non-hypertensive and non-diabetic individuals: the Framingham Heart Study. Circulation 2005;112:969-975.

2 Yan S, Yao F, Huang L, Ruan Q, Shen X, Zhang S, Huang C: Low-grade albuminuria associated with subclinical left ventricular diastolic dysfunction and left ventricular remodeling. Exp Clin Endocrinol Diabetes 2015;123:515-523.

Tanaka F, Komi R, Makita S, Onoda T, Tanno K, Ohsawa M, Itai K, Sakata K, Omama S, Yoshida Y, Ogasawara K, Ishibashi Y, Kuribayashi T, Okayama A, Nakamura M; Iwate-Kenco Study Group: Low-grade albuminuria and incidence of cardiovascular disease and all-cause mortality in nondiabetic and normotensive individuals. J Hypertens 2016;34:506-512.

Levey AS, Stevens LA, Schmid CH, Zhang YL, Castro AF 3rd, Feldman HI, Kusek JW, Eggers P, Van Lente F, Greene T, Coresh J; CKD-EPI (Chronic Kidney Disease Epidemiology Collaboration): A new equation to estimate glomerular filtration rate. Ann Intern Med 2009;150:604-612.

5 National Health Information Portal: Alcohol drinking. Sejong: Ministry of Health and Heath. Available from: URL: http://health.cdc.go.kr/health/HealthInfoArea/HealthInfo/View. do?idx=14520 (Accessed on May 23th 2017). 


\section{Kidney \\ Blood Pressure Research}

\begin{tabular}{l}
\hline Kidney Blood Press Res 2017;42:338-346 \\
\begin{tabular}{l|l}
\hline DOI: 10.1159/000477784 & $\begin{array}{l}\text { @ } 2017 \text { The Author(s). Published by S. Karger AG, Basel } \\
\text { www.karger.com/kbr }\end{array}$ \\
\hline Published online: June 08, 2017 &
\end{tabular} \\
\hline
\end{tabular}

Kang et al.: Periodontitis and High UACR1

16 Grundy SM, Cleeman JI, Daniels SR, Donato KA, Eckel RH, Franklin BA, Gordon DJ, Krauss RM, Savage PJ, Smith SC Jr, Spertus JA, Costa F; American Heart Association.; National Heart, Lung, and Blood Institute: Diagnostic and management of the metabolic syndrome: an American Heart Association/National Heart, Lung, and Blood Institute Scientific Statement. Circulation 2005;112:2735-2752.

$>17$ Hong JW, Noh JH, Kim DJ: The prevalence and associated factors of periodontitis according to fasting plasma glucose in the Korean adults: The 2012-2013 Korea National Health and Nutrition Examination Survey. Medicine 2016;95:e3226.

-18 Preshaw PM, Alba AL, Herrera D, Jepsen S, Konstantinidis A, Makrilakis K, Taylor R: Periodontitis and diabetes: a two-way relationship. Diabetologia 2012;55:21-31.

19 Fisher MA, Taylor GW, West BT, McCarthy ET: Bidirectional relationship between chronic kidney and periodontal disease: a study using structural equation modeling. Kidney Int 2011;79:347-355.

20 Schenkein HA, Loos BG: Inflammatory mechanisms linking periodontal diseases to cardiovascular diseases. J Clin Periodontol 2013;40:S51-S69.

-21 Seinost G, Wimmer G, Skerget M, Thaller E, Brodmann M, Gasser R, Bratschko RO, Pilger E: Periodontal treatment improves endothelial dysfunction in patients with severe periodontitis. Am Heart J 2005;149:1050-1054.

22 DeStefano F, Anda RF, Kahn HS, Williamson DF, Russell CM: Dental disease and risk of coronary heart disease and mortality. BMJ 1993;306:688-691.

-23 Ajwani S, Mattila KJ, Närhi TO, Tilvis RS, Ainamo A: Oral health status, C-reactive protein and mortality-a 10 year follow-up study. Gerodontology 2003;20:32-40.

24 Ajwani S, Mattila KJ, Tilvis RS, Ainamo A: Periodontal disease and mortality in an aged population. Spec Care Dentist 2003;23:125-130.

-25 Kshirsagar AV, Craig RG, Moss KL, Beck JD, Offenbacher S, Kotanko P, Klemmer PJ, Yoshino M, Levin NW, Yip JK, Almas K, Lupovici EM, Usvyat LA, Falk RJ: Periodontal disease adversely affects the survival of patients with end-stage renal disease. Kidney Int 2009;75:746-751.

-26 Fisher MA, Taylor GW, Papapanou PN, Rahman M, Debanne SM: Clinical and serologic markers of periodontal infection and chronic kidney disease. J Periodontol 2008;79:1670-1678.

27 Chen LP, Chiang CK, Peng YS, Hsu SP, Lin CY, Lai CF, Hung KY: Relationship between periodontal disease and mortality in patients treated with maintenance hemodialysis. Am J Kidney Dis 2011;57:276-282.

28 Siribamrungwong M, Yothasamutr K, Puangpanngam K: Periodontal treatment reduces chronic systemic inflammation in peritoneal dialysis patients. Ther Apher Dial 2014;18:305-308.

29 Han K, Nam GE, Kim DH, Park JB, Ko Y, Roh YK, Cho KH, Park YG: Association of periodontitis with urinary albumin excretion in Korean adults with diabetes: The 2012 Korea National Health and Nutrition Examination Survey. Medicine (Baltimore) 2015;94:e1839.

30 Kim Y, Park S, Kim NS, Lee BK: Inappropriate survey design analysis of the Korean National Health and Nutrition Examination Survey may produce biased results. J Prev Med Public Health 2013;46:96-104.

- 31 Hong JW, Ku CR, Noh JH, Ko KS, Rhee BD, Kim DJ: Association between low-grade albuminuria and cardiovascular risk in Korean adults: the 2011-2012 Korea National Health and Nutrition Examination Survey. PLoS One 2015;10:e0118866.

-32 Zhang J, Chen Y, Xu Y, Li M, Wang T, Xu B, Sun J, Xu M, Lu J, Bi Y: Low-Grade Albuminuria is associated with metabolic syndrome and its components in middle-aged and elderly Chinese population. PLoS One 2013;8:e65597. 